Pulmoner Hipertansiyonlu Hastalarımızın Klinik, Epidemiyolojik ve

Hemodinamik Verilerinin Değerlendirilmesi

Evaluation of Clinical, Epidemiological and Hemodynamic Data of Our

Patients with Pulmonary Hypertension

Dolunay Gürses, Özlem Gül

Pamukkale Üniversitesi Tıp Fakültesi, Çocuk Kardiyoloji Bilim Dalı, Denizli

$\ddot{O} Z$

GİRIŞ ve AMAÇ: Bu çalışma, pulmoner arteriyel hipertansiyon (PAH) tanılı hastalarımızın epidemiyolojik özelliklerinin, yaşam kalitelerinin, tedavi yöntemlerinin ve etkinliğinin belirlenmesi amaciyla yapıld1.

YÖNTEM ve GEREÇLER: Ocak 2008-Aralık 2016 tarihleri arasında Çocuk Kardiyoloji bölümünde PAH nedeniyle izlenen 15 hasta (11 kız, 4 erkek) retrospektif olarak değerlendirildi.

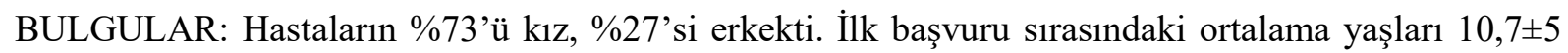
(3-20) yıldı. Hastaların sadece birinde (\%7) primer PAH, iki olguda (\%13) kronik akciğer hastalı̆̆ına sekonder PAH mevcuttu. On iki hastada (\%80) doğumsal kalp hastalığına sekonder PAH saptandı. Bu hastalardan 10 tanesi Eisenmenger sendromuyken, iki hastada kapatılmış sol-sağ şantlı kalp hastalığ1 mevcuttu. Eisenmenger sendromu olan 10 hastanın altısında (\%60) VSD, ikisinde (\%20) atriyoventriküler septal defekt mevcuttu. En sık görülen semptom çabuk yorulma (\%83) idi. Hastaların tamamına telekardiyografi, elektrokardiyogram (EKG) ve ekokardiyografi yapılmış olup, \%87'sine altı dakika yürüme testi ve anjiyografi yapılmıştı. Anjiyografi yapılan hastaların hepsine vazoreaktivite testi yapılmıştı ve hepsinde negatif saptanmıştı. Hastaların son kontrollerindeki tedavileri değerlendirildiğinde; \%53,3'ünün monoterapi, \%46,7'sinin kombine tedavi aldığı görüldü. En s1k başlanan monoterapi bosentandı (\%46,7). Dört hastamız izlemde kaybedilmişti.

TARTIŞMA ve SONUÇ: Pulmoner arteriyel hipertansiyon birçok etiyolojik nedene bağlı oluşabilen kronik ilerleyici bir hastalıktır. Tedavi edilmezse; pulmoner vasküler rezistansta progresif artış sonucunda sağ ventrikül yetmezliği ve erken ölüme neden olur. PAH şüphesi olan hastaların deneyimli merkezlere yönlendirilmeleri, uygun tanı, takip ve tedavi seçeneklerinin kullanılmasını sağlayacaktır.

Anahtar Kelimeler: Eisenmenger sendromu, pulmoner hipertansiyon, tedavi.

Türkçe Kısa Başlık: Pulmoner hipertansiyonlu hastaların değerlendirilmesi

Yayın hakları Güncel Pediatri'ye aittir. 


\title{
Evaluation of Clinical, Epidemiological and Hemodynamic Data of Our Patients With Pulmonary Hypertension
}

\author{
Dolunay Gürses, Özlem Gül \\ Department of Pediatric Cardiology, Pamukkale University, Denizli, Turkey
}

\section{ABSTRACT}

INTRODUCTION: This study was conducted to determine the epidemiological characteristics, quality of life, treatment modalities and efficacy of our treatment in patients with pulmonary arterial hypertension $(\mathrm{PAH})$.

METHODS: Fifteen patients (11 girls, 4 boys) who were followed up for PAH in the Department of Pediatric Cardiology between January 2008 and December 2016 were evaluated retrospectively.

RESULTS: The mean age of patients at first admission was 10.7 5 (3-20) years. Only one of the patients (7\%) had primary PAH, and two patients (13\%) had PAH associated with chronic lung disease. Twelve patients (80\%) had PAH secondary to congenital heart disease. Of these patients, 10 had Eisenmenger syndrome and two were operated for congenital heart disease with a left to right shunt. Six patients (60\%) had VSD, and two (20\%) had atrioventricular septal defect in 10 patients with Eisenmenger syndrome. The most common symptom was easy fatigue (83\%). All of the patients had telecardiography, electrocardiogram and echocardiography, and $87 \%$ of patients underwent six minute walking test and angiography. All patients undergoing angiography were tested for vasoreactivity and all were negative. When the treatments at the last controls of the patients are evaluated; $53.3 \%$ of the patients received monotherapy, $46.7 \%$ received combination therapy. The most frequently started monotherapy was bosentan (46.7\%). Four patients were died during follow-up.

DISCUSSION and CONCLUSION: Pulmonary arterial hypertension is a chronic progressive disease that can be caused by many etiologic factors. If not treated; progressive increase in pulmonary vascular resistance leads to right ventricular failure and premature death. Accumulating PAH patients in experienced centers will ensure appropriate diagnosis, follow-up and treatment options.

Keywords: Eisenmenger syndrome, pulmonary hypertension, treatment İngilizce Kısa Başlık: Evaluation of patients with pulmonary hypertension 


\section{Giriş}

Pulmoner arteriyel hipertansiyon $(\mathrm{PAH})$; term bebeklerde deniz seviyesinde postnatal üçüncü aydan sonra her iki akciğerin tüm segmentlerine eşit kan akımı dağılımı varken ortalama pulmoner arter basıncının $\geq 25 \mathrm{mmHg}$ olması olarak tanımlanır (1). Çocukluk döneminde herhangi bir yaşta ortaya çıkabilir. Çocuklarda PAH için en son tanısal sınıflandırma 2013'de Nice'de (Fransa) düzenlenen 5. Dünya Pulmoner Hipertansiyon Sempozyumu'nda bildirilmiştir (2). PAH sınıflaması tekrar düzenlenmiş ve pediatrik yaş grubundan başlayan erişkin yaşa ulaşan doğumsal kalp hastalıklarına yaklaşımı kolaylaştırmak amaçlanmıştır. Ayrıca doğuştan kalp hastalıkları (DKH) ile birlikte olan PAH sınıflandırmasında da değişiklik yapılmıştır. Sağdan sola şantla seyreden Eisenmenger sendromu, opere edilmiş veya edilmemiş soldan sağa şantlar, küçük deliklerle birlikte olan rastlantısal PAH ve opere edilmiş fakat pulmoner hipertansiyonu devam eden veya artan hastalar olarak dört tip olarak tanımlanmıştır.

Çocukluk çağında PAH nedenleri yetişkinlerden farklılık gösterir. İdiyopatik PAH çocuklarda \%40'a varan oranlarda görülürken, DKH'ya bağlı pulmoner hipertansiyon oranı \%50'leri bulmaktadır. Ventriküler septal defekt (VSD), atriyoventriküler septal defekt (AVSD), patent duktus arteriyozus (PDA), atriyal septal defekt (ASD), büyük arter transpozisyonu, trunkus arteriyozus, total anormal pulmoner venöz dönüş anomalisi, çift çıkışlı sağ ventrikül gibi doğumsal kalp hastalıkları PAH’a neden olabilir (3). Artmış pulmoner kan akımı ve basıncı pulmoner damar yatağındaki değişikliklerden sorumlu tutulmakla birlikte mekanizma tam olarak anlaşılamamıştır (4).

$\mathrm{Bu}$ çalışma ile PAH tanılı hastalarımızın demografik, etiyolojik, klinik, laboratuvar verileri, tedavi yöntemleri ve tedavi etkinliği ile ilgili bulgular ve deneyimlerimiz aktarılmaya çalışılmıştır.

\section{Gereç ve Yöntemler}

Pamukkale Üniversitesi Tıp Fakültesi Çocuk Kardiyoloji Bilim Dalı'nda Ocak 2008-Aralık 2016 yılları arasında PAH nedeniyle izlenen 15 hastanın kayıtları retrospektif olarak değerlendirildi. Çalışmaya alınan hastaların demografik özellikleri, yakınmaları, "New York Heart Association" 
(NYHA) fonksiyonel sınıfı, fizik bakı bulguları, tanı yaşı, kalp hastalığı öyküsü, pulmoner hipertansiyon nedeni, altı dakika yürüme testi, elektrokardiyografi, ekokardiyografi ve kalp kateterizasyonu verileri dosyalarından elde edildi. Ortalama pulmoner arter basıncı pulse dalga Doppler ekokardiyografi ile ölçülen pulmoner hız akselerasyon zamanı (pulmoner kapaktan geçen akımın başlangıcından tepe noktasına kadar geçen süre) kullanılarak 79-(0.45 x pulmoner hız akselerasyon zamanı) formülü ile hesaplandı (5). Olgulara uygulanan tedaviler ve izlemlerine ait bilgiler değerlendirildi. Çalışma için hastane yerel etik kurulundan onay alınd1.

Verilerin istatistiksel değerlendirmesi Statistical Package Social Sciences for Windows (SPSS Inc., Chicago) 18.0 programında yapıldı, tanımlayıcı istatistiklerin yanında ki-kare testi kullanıldı. Sonuçlar ortalama \pm standart sapma olarak verildi. $\mathrm{P}<0,05$ değeri istatistiksel anlamlılık sınırı olarak kabul edildi.

\section{Bulgular}

Çalışmaya alınan 15 PAH'lı hastanın 11'i kız (\%73), 4'ü erkek (\%27) idi (p>0,05). Hastaların

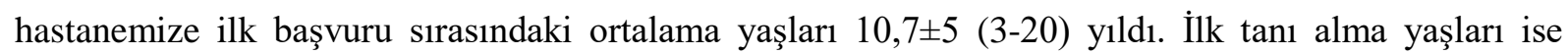
ortalama $6 \pm 4$ (1-14) y1l olarak saptand. İki olgumuzda Down sendromu vardı.

Hastalarda en sık görülen yakınma çabuk yorulma (\%83) idi. Hastaların \%73'ünde dispne \%13'ünde çarpıntı ve \%7'sinde hemoptizi mevcuttu. Fonksiyonel sınıf kapasite değerlendirilmesi yapıldığında; \%75'i NYHA fonksiyonel sınıf III kapasiteye, \%25'i NYHA fonksiyonel sinıf II kapasiteye sahipti. Hastaların \%73'ünde çomak parmak mevcuttu.

Hastaların sadece birinde (\%7) primer PAH vardı. İki olguda (\%13) kronik akciğer hastalığına sekonder PAH gelişmişti. On iki hastada ise $(\% 80)$ doğumsal kalp hastalığına sekonder PAH vardı. Doğumsal kalp hastalığı saptanan 12 olgunun 2'si (\%16) erkek, 10’u (\%84) kızdı. Bu hastalardan 10 tanesi (\%84) Eisenmenger sendromu iken, iki hastada kapatılmış sol-sağ şantlı kalp hastalığı mevcuttu. $\mathrm{Bu}$ hastaların birinde PDA cihazla kapatılmış, diğerinde ise VSD cerrahi olarak kapatılmıştı. Eisenmenger sendromu olan 10 hastanın 6'sında (\%60) VSD, 2'sinde (\%20) AVSD, 2'sinde (\%20) çift 
çıkışlı sağ ventrikül+büyük arter transpozisyonu mevcuttu. Hastalara ilişkin tanısal veriler Tablo I'de verilmiştir.

Tablo I: Pulmoner Hipertansiyonlu Hastalarımızın Tanısal Verileri

\begin{tabular}{|c|c|c|c|c|}
\hline VAKA & Tanı Yaşı & Başvuru yaşı & Cinsiyet & PAH etiyolojisi \\
\hline Vaka 1 & 3 yaş & 20 yaş & $\mathrm{E}$ & DORV+D-TGA \\
\hline Vaka 2 & 1 yaş & 8 yaş & $\mathrm{K}$ & DORV+D-TGA \\
\hline Vaka 3 & 11 yaş & 9 yaş & K & VSD \\
\hline Vaka 4 & 6 yaş & 15 yaş & K & AVSD \\
\hline Vaka 5 & 6 yaş & 17 yaş & K & VSD (Opere) \\
\hline Vaka 6 & 14 yaş & 16 yaş & K & PDA (Cihazla kapatılmış) \\
\hline Vaka 7 & 14 yaş & 14 yaş & K & VSD \\
\hline Vaka 8 & 1 yaş & 8 yaş & K & VSD \\
\hline Vaka 9 & 4 yaş & 12 yaş & $\mathrm{K}$ & VSD \\
\hline Vaka 10 & 2 yaş & 5 yaş & K & VSD \\
\hline Vaka 11 & 4 yaş & 5 yaş & $\mathrm{K}$ & AVSD \\
\hline Vaka 12 & 6 yaş & 6 yaş & K & Primer PAH \\
\hline Vaka 13 & 6 yaş & 13 yaş & $\mathrm{E}$ & VSD \\
\hline Vaka 14 & 3 yaş & 3 yaş & $\mathrm{E}$ & Kronik akciğer hastalığı (Bronkopulmoner displazi) \\
\hline Vaka 15 & 10 yaş & 10 yaş & $\mathrm{E}$ & Kronik akciğer hastalığ 1 (Kistik fibrozis) \\
\hline
\end{tabular}

Hastaların tamamına telekardiyografi, elektrokardiyogram ve ekokardiyografi yapılmıştı. Elektrokardiyografik değerlendirmede hastaların tamamında sağ aks sapması, sağ ventrikül hipertrofisi, P pulmonale ve sağ dal bloğu mevcuttu. İki hastada geçirilmiş supraventriküler taşikardi atağı öyküsü vard1. Transtorasik ekokardiyografik olarak değerlendirilen ortalama pulmoner arter basıncı (PAB) 47,6 $7,4 \mathrm{mmHg}$ idi. Hastaların on üçüne (\%87) altı dakika yürüme testi ve anjiyografi yapılmıştı. İki hastada ise genel durum bozukluğu nedeniyle yapılamamıştı. Anjiyografi yapılan hastaların hepsine vazoreaktivite testi yapılmış ve hepsinde negatif saptanmıştı. Hastaların başvuru sırasındaki altı dakika 


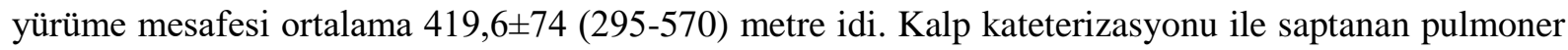

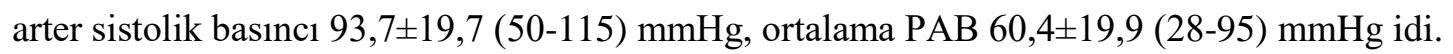

Hastaların izlem süresi ortalama 52 $\pm 30,4$ (10-100) ay olarak saptandı. Dört hastamız izlemde kaybedildi. Bu olguların birisi primer PAH tanısı ile izleminin dokuzuncu ayında, birisi kronik akciğer hastalığına bağlı pulmoner hipertansiyon nedeniyle izleminin birinci yılında, diğer ikisi ise doğumsal kalp hastalığına bağlı Eisenmenger sendromu nedeniyle izlemlerinin dördüncü ve beşinci yıllarında kaybedildi. Sağ kalan hastalarımızın son kontrollerinde altı dakika yürüme mesafesi 464,4 $\pm 70,7$ (380590) metre idi. Son kontroldeki altı dakika yürüme mesafesi, başvuru sırasına göre artmış olmakla birlikte farklılık istatistiksel olarak anlamlı değildi $(\mathrm{p}>0,05)$. Hastaların son kontrollerindeki tedavileri değerlendirildiğinde; \%53,3’ünün monoterapi, \%46,7'sinin kombine tedavi aldığı görüldü. En s1k başlanan monoterapi bosentandı $(\% 46,7)$. Kombine tedavi alan hastalar ise bosentan+tadalafil $(\% 6,7)$, bosentan+ilioprost $(\% 6,7)$, bosentan+ilioprost+sildenafil $(\% 13,3)$, masitentan+ilioprost+tadalafil $(\% 6,7)$ bosentan+ilioprost+tadalafil $(\% 13,3)$ tedavileri almaktaydı. İzlemde 18 yaşını dolduran dört hastamızdaki sildenafil tedavisi tadalafil ile, bir hastamızdaki bosentan tedavisi de masitentan ile değiştirilmişti.

\section{Tartışma}

Pulmoner hipertansiyon; kardiyak ve nonkardiyak sebeplere bağlı olarak pulmoner vasküler rezistans ve pulmoner arter basıncının progresif artışı ile giden nadir bir hastalıktır. Tedavi edilmediğinde pulmoner vasküler yatakta yeniden yapılanma, sağ kalp yetmezliği ve ölümle sonuçlanır $(3,6,7)$. Pulmoner arter basıncının temel belirleyicileri pulmoner vasküler rezistans ve pulmoner arter kan akımıdır. Doğumdan hemen sonra PVR 8-10 WU/m² düzeyinde iken, haftalar içinde düşerek postnatal 6-8. haftada normal erişkin düzey olan 1-3 WU/m²'ye ulaşır. Pulmoner vasküler rezistansın 3 WU $/ \mathrm{m}^{2}$ 'den yüksek olması durumu belirgin PAH olarak kabul edilmektedir (8).

Pulmoner hipertansiyonun; dünya üzerinde 25 milyondan fazla bireyi etkilediği ve çocukluk çağında prevelansının yaklaşık bir milyonda 10-20 olduğu tahmin edilmektedir (9). Çocukluk çağında 
hastalık her iki cinsi eşit olarak etkilerken; puberteden sonra kadınlarda daha yaygın görüldüğü bildirilmiştir $(3,4)$. Çalışmamızda da PAH tanılı olgularımızın çoğunluğu kızdı.

Çocuklarda genellikle doğumsal kalp hastalığı, nadiren parankimal akciğer hastalığı, kollajen doku ve diğer hastalıklar PAH'a yol açmaktadır. İdiyopatik pulmoner arteriyel hipertansiyon (IPAH) çocukluk çağında nadirdir ve genellikle genç kadınlarda görülür $(1,8)$. Doğumsal kalp hastalığına bağl1 PAH içinde en büyük grubu asiyanotik, ikinci grubu ise siyanotik DKH'a bağlı PAH oluşturmaktadır. Sol-sağ şantlar ve basınç artışı nedeniyle pulmoner yatağın uzun süre yüksek kan akışına maruz kalması pulmoner obstrüktif arteriyopatiye ve sonuçta pulmoner vasküler direnç artışına neden olabilir. Pulmoner vasküler direnç sistemik vasküler dirence yaklaşır ya da onu geçerse şant tersine döner; bu durum Eisenmenger sendromu olarak adlandırılır (8). Hastalarımızın \%80'inde doğumsal kalp hastalıklarına bağlı PAH mevcut olup bu hastalarımızın da\%84'ünde Eisenmenger sendromu vardı. Sadece bir olgumuzda primer PAH ve iki olgumuzda ise kronik akciğer hastalığına sekonder PAH mevcuttu.

Pulmoner hipertansiyon gelişen hastaların önemli bir kısmında efor kapasitesinde kısıtlanma, nefes darlığı, halsizlik, göğüs ağrısı, bayılma hissi, periferik ödem, çarpıntı gibi yakınmalar mevcuttur. $\mathrm{Bu}$ hastalarda siyanoz, çomak parmak, senkop, hemoptizi, serebrovasküler olaylar, pıhtılaşma anormallikleri ve ani ölüm görülebilir (8). Hastalarımızda da en sık görülen yakınma çabuk yorulma, efor kapasitesinde kısıtlanma ve dispneydi. Bir hastamızda ise hemoptizi yakınması mevcuttu.

Pulmoner hipertansiyon tanısında detaylı bir öykü ve fizik muayeneyi takiben öncelikle noninvaziv yöntemlerle hastalığın varlığı araştırılmalıdır. Etiyolojik sebeplere yönelik elektrokardiyografi, telekardiyografi, transtorasik ekokardiyografi, laboratuvar tetkikleri ve solunum fonksiyon testleri yapılmalıdır. Ventilasyon/perfüzyon sintigrafisi özellikle kronik tromboembolik pulmoner hipertansiyon tanısı için değerli olup, bilgisayarlı tomografi ve manyetik rezonans görüntüleme gibi tetkikler ile de trombüsler ve proksimal pulmoner arterler görüntülenebilir $(3,6)$. Ayrıca PAH'da pulmoner arter basıncının, pulmoner vasküler direncin ve pulmoner vazoreaktivitenin belirlenmesinde kardiyak kateterizasyon oldukça önemlidir. 
Vazoreaktivite testinin negatif olması kalıcı vasküler obstrüktif hastalık tanısını kesinleştirir $(1,3,6)$. Hastalarımızın hepsinde vazoreaktivite testi negatifti.

Pulmoner arteriyel hipertansiyon hemodinamik birçok mekanizmanın ortak sonucu gelişir. Vazokonstriksiyon, arter duvarında yeniden biçimlenme (remodeling) ve in situ tromboz pulmoner arter lümenini daraltarak PAH'a neden olur. Pulmoner damar duvar hasarı sonucunda vazokonstriktif mediyatörlerle (endotelin-1), vazodilatör mediyatörler (prostasiklin, nitrik oksit) arasındaki denge bozulur (8). PAH'da etiyoloji farklı olsa bile, tedavi stratejilerinde üç ana yol üzerine hedeflenilmektedir. Tedavide endotelin reseptör antagonistleri, prostasiklin ve prostasiklin analogları ile fosfodiesteraz tip-5 inhibitörleri üç ana grubu oluşturmaktadır (10). Randomize, çok merkezli, çift-kör, plasebo kontrollü BREATHE-5 çalışmasında (11), fonksiyonel kapasitesi sınıf III olan Eisenmenger sendromlu hastalarda 16 haftalık bosentan tedavisinin iyi tolere edildiği ve periferal oksijen satürasyonunu bozmadan 6 dakika yürüme testini iyileştirdiği gösterilmiştir. Pulmoner arteriyel hipertansiyonda kullanılan diğer bir ilaç grubu fosfodiesteraz tip-5 inhibitörleridir. Fosfodiesteraz inhibitörü olan sildenafil etkisini hücre içindeki siklik GMP düzeyini artırarak gösterir (12). Pulmoner arteriyel hipertansiyonlu hastalarda prostasiklin sentezi azalmış olup; bu durum vazodilatasyon ve antiproliferatif özelliklere sahip prostaglandin $\mathrm{I}_{2}$ 'nin yetersiz üretimine neden olur. Prostasiklin analoglarından epoprostenol, treprostinil ve ilioprost İPAH ve doğumsal kalp hastalığıyla ilişkili PAH'da kullanılabilmektedir. Ortalama yaşları 15 yıl olan 20 hastanın alındığ bir çalışmada, bir yıl süreyle $\mathrm{PGI}_{2}$ kullanımının hemodinamiyi ve hayat kalitesini artırdığı gösterilmiştir (13). Hastalarımızın $\% 93,3$ 'ü endotelin reseptör antagonisti olan bosentan veya masitentan alırken, \%40'1 prostasiklin analoğu olan ilioprost ve \%46,6's1 fosfodiesteraz inhibitörleri olan sildenafil ya da tadalafil kullanmaktaydı. PAH klavuzlarında vazoreaktivite testi pozitif hastalarda, kalsiyum kanal blokerleri ilk seçenek tedavi ajanı olarak yerini almıştır. Vazoreaktivite testi negatif olan düşük ve orta riskli hastalarda endotelin reseptör antagonistleri ya da fosfodiesteraz inhibitörlerinin monoterapi olarak seçilebileceği, yüksek riskli grupta ise prostasiklin antagonistleri ve erken kombine tedavinin başlanması gerektiği belirtilmektedir (14). Hastalarımızın da \%53,3’ü monoterapi, \%46,7'si kombine tedavi alıyordu ve monoterapide en s1k kullanılan ajan bosentandı. 
Doğumsal kalp hastalıklarının uygun şekilde izlemi ve cerrahi tedavi zamanlamasının doğru yapılması pulmoner hipertansiyon gelişiminin önlenmesinde oldukça önemlidir. Pulmoner hipertansiyon tedavisi ve izlemi tecrübeli merkezlerde dikkatli bir şekilde yapılmalıdır. Son yıllarda PAH ile ilgili gelişmeler bu hastalar için daha uzun ve kaliteli bir hayat sağlanabilmesi olanağını sunmaktadir.

Çıkar çatışması: Yazarlar ya da çalışma sonuçları ile ilgili herhangi bir çıkar çatışması yoktur.

\section{Kaynaklar}

1. Ivy DD, Abman SH, Barst RJ, Bonnet D, Fleming TR, Haworth SG, et al. Pediatric pulmonary hypertension. J Am Coll Cardiol 2013;62(25):117-26.

2. Simonneau G,Gatzoulis MA, Adatia I, Celermajer D, Denton C, Ghofrani A, et al. Updated clinical classification of pulmonary hypertension. J Am Coll Cardiol 2013;62(25):34-41.

3. Olguntürk R. Pediatrik pulmoner hipertansiyon ve doğumsal kalp hastalıklarına bağlı pulmoner arteriyel hipertansiyon. Anadolu Kardiyol Derg 2010; Özel Sayı 1:50-6.

4. Abman SH, Hansmann G, Archer SL, Ivy DD, Adatia I, Chung WK, et al. Pediatric Pulmonary Hypertension: Guidelines from the American Heart Association and American Thoracic Society. Circulation 2015;132(21):2037-99.

5. Özben B, Başaran Y. Pulmoner arteriyel hipertansiyonda ekokardiyografi ve diğer görüntüleme yöntemleri. Anadolu Kardiyol Derg 2010;10 (1):27-35.

6. Hansmann G, Apitz C, Abdul-Khaliq H, Alastalo TP, Beerbaum P, Bonnet D, et al. Executive summary. Expert consensus statement on the diagnosis and treatment of paediatric pulmonary hypertension. The European Paediatric Pulmonary Vascular Disease Network, endorsed by ISHLT and DGPK. Heart 2016;102(Suppl. 2):ii86-ii100.

7. Chen IC, Dai ZK. Insight into pulmonary arterial hypertension associated with congenital heart disease (PAH-CHD): Classification and pharmacological management from a pediatric cardiological point of view. Acta Cardiol Sin 2015;31(6):507-15. 
8. Humpl T, Ingram SN. Pulmonary vascular disease. In Anderson R, Baker EJ, Penny D, Redington AN, Rigby ML, Wernovsky G (eds). Pediatric Cardiology. 3rd edition. Churchill Livingstone Publication; 2010.p.1147.

9. Elliott CG, Barst RJ, Seeger W, Porres-Aguilar M, Brown LM, Zamanian RT, et al. World wide physician education and training in pulmonary hypertension: pulmonary vascular disease: the global perspective. Chest 2010;137(6):85-94.

10. Gatzoulis MA, Alonso-Gonzalez R, Beghetti M. Pulmonary arterial hypertension in paediatric and adult patients with congenital heart disease. Eur Respir Rev 2009;18(113):154-61.

11. Galiè N, Beghetti M, Gatzoulis MA, Granton J, Berger RM, Lauer A, et al. Bosentan Randomized Trial of Endothelin Antagonist Therapy-5 (BREATHE-5) Investigators. Bosentan therapy in patients with Eisenmenger syndrome: a multicenter, double-blind, randomized, placebo-controlled study. Circulation 2006;114(1):48-54.

12. Y1ldız M, Sunar H, Esen AM. Treatment strategies of pulmonary arterial hypertension associated with congenital heart defects with shunt: A group of important intensive care patient in cardiology and cardiovascular surgery. Kosuyolu Kalp Derg 2013;16(3):173-6.

13. Rosenzweig EB, Kerstein D, Barst RJ. Long-term prostacyclin for pulmonary hypertension with associated congenital heart defects. Circulation 1999;99(14):1858-65.

14. Galie N, Humbert M, Vachiery JL, Gibbs S, Lang I, Torbicki A, et al. 2015 ESC/ERS Guidelines for the diagnosis and treatment of pulmonary hypertension: The Joint Task Force for the Diagnosis and Treatment of Pulmonary Hypertension of the European Society of Cardiology (ESC) and the European Respiratory Society (ERS): Endorsed by: Association for European Paediatric and Congenital Cardiology (AEPC), International Society for Heart and Lung Transplantation (ISHLT). Eur Heart J 2016;37(1):67-119. 